\title{
Synchronization in Tempered Fractional Complex Networks via Auxiliary System Approach
}

\author{
Weiyuan Ma $\mathbb{D}^{1},{ }^{1}$ Changpin $L i \mathbb{D}^{2}$ and Jingwei Deng $\mathbb{D}^{1}$ \\ ${ }^{1}$ School of Mathematics and Computer Science, Northwest Minzu University, Lanzhou 730000, China \\ ${ }^{2}$ Department of Mathematics, Shanghai University, Shanghai 200444, China \\ Correspondence should be addressed to Changpin Li; lcp@shu.edu.cn
}

Received 29 October 2018; Revised 25 January 2019; Accepted 26 September 2019; Published 25 November 2019

Academic Editor: Thierry Floquet

Copyright (c) 2019 Weiyuan Ma et al. This is an open access article distributed under the Creative Commons Attribution License, which permits unrestricted use, distribution, and reproduction in any medium, provided the original work is properly cited.

In the famous continuous time random walk (CTRW) model, because of the finite lifetime of biological particles, it is sometimes necessary to temper the power law measure such that the waiting time measure has a convergent first moment. The CTRW model with tempered waiting time measure is the so-called tempered fractional derivative. In this article, we introduce the tempered fractional derivative into complex networks to describe the finite life span or bounded physical space of nodes. Some properties of the tempered fractional derivative and tempered fractional systems are discussed. Generalized synchronization in two-layer tempered fractional complex networks via pinning control is addressed based on the auxiliary system approach. The results of the proposed theory are used to derive a sufficient condition for achieving generalized synchronization of tempered fractional networks. Numerical simulations are presented to illustrate the effectiveness of the methods.

\section{Introduction}

In the past few decades, the study of fractional calculus has attracted substantial attention. Fractional calculus is a powerful mathematical tool for modeling systems in the fields of secure communication, biological science, chemical reactors, laser systems, and so on [1-5]. Recently, tempered fractional derivatives have drawn the wide interests of researchers. A tempered fractional derivative is closer to reality in the sense of the finite life span or bounded physical space of particles. As a generalization of fractional calculus, tempered fractional calculus does not simply have the properties of fractional calculus but can describe some other complex dynamics [6,7]. Tempered fractional derivatives and the corresponding tempered fractional differential equations have played key roles in poroelasticity [8], finance [9], ground-water hydrology [10], geophysical flows [11], and so on. Therefore, complex network models with tempered fractional dynamics can become more realistic.

Synchronization, as one of the vital dynamical phenomena, exists not only in integer systems but also in fractional systems. Its applications range from electrology to biology, from physics to engineering, and even from economics to nervous system, communication system, and control processing [12-16]. Many studies have focused on the outer synchronization between two fractional complex networks [17-19]. Yu et al. investigated $\alpha$-synchronization for fractional neural networks [20]. Wu et al. constructed outer synchronization for two different fractional complex networks with nonlinear controllers [21]. Yang and Jiang considered adaptive synchronization for fractional complex networks with uncertain parameters [22]. Chai et al. proposed a pinning synchronization for general fractional complex networks [23]. Dadras et al. studied fractional integral sliding-mode control for uncertain fractional nonlinear systems [24]. Shao et al. discussed adaptive slidingmode synchronization for a class of fractional chaotic systems [25]. However, in most of this work, all individuals in two networks are assumed to have completely identical dynamics, which is not the case in real practice, for example, predatorprey interactions in ecological communities. Clearly, groups of predators and prey have their own dynamical behavior. Therefore, outer synchronization between two different tempered fractional complex networks is a practical and significant problem worth investigating. 
The scenario in which two networks reach harmonious coexistence is regarded as generalized synchronization, which is weaker than complete synchronization [26]. Generalized synchronization exists widely in nature and society. For example, predators and preys influence each other's behaviors. Predators cannot live without preys, and too many predators would bring the preys into extinction. The complex systems of predators and preys will finally reach harmonious coexistence without man-made sabotage. The auxiliary system approach [27] was proposed to realize generalized synchronization between two complex networks. In addition to the drive and response systems, this method constructs an auxiliary system that has an identical dynamical system to that of the response system, as described by

$$
\begin{cases}{ }_{C} D_{0, t}^{\alpha_{1}, \lambda_{1}} x(t)=G(x(t)), & \text { (drive system), } \\ { }_{C} D_{0, t}^{\alpha_{2}, \lambda_{2}} y(t) H(x(t), y(t)), & \text { (response system), } \\ { }_{C} D_{0, t}^{\alpha_{2}, \lambda_{2}} z(t)=H(x(t), z(t)), & \text { (auxiliary system), }\end{cases}
$$

where $x(t), y(t), z(t) \in \mathbb{R}^{n}$ are the states of drive, response, and auxiliary system, respectively. ${ }_{C} D_{0, t}^{\alpha, \lambda}$ is the tempered fractional Caputo derivative operator which is defined later on. If the response system and auxiliary system reach complete synchronization, that is, $\lim _{t \longrightarrow \infty}\|y(t)-z(t)\|=0$ for any initial conditions $y\left(t_{0}\right) \neq z\left(t_{0}\right)$, then generalized synchronization between the drive system and response systems is achieved. Figure 1 gives the schematic diagram of generalized synchronization based on the auxiliary system approach. Specifically, layer I is the drive system, and layer II is the response system which is driven by signals from layer I. Layer III is the auxiliary system, which is an identical duplication of layer II driven by the same signals from the drive layer. This technique is a very effective method to realize outer synchronization between two complex networks [29]. However, as far as we know, no one has discussed the auxiliary system approach for fractional complex networks.

Synchronization of tempered fractional complex networks in a generalized sense leads to richer behavior than identical node dynamics in coupled networks. It may disclose a more complicated connection between the synchronized trajectories in the state spaces of coupled networks. In this paper, we first study the tempered fractional complex network and its generalized synchronization. Additionally, we derive some properties of tempered fractional calculus to construct the synchronization criteria of tempered fractional complex networks. Tempered MittagLeffler stable is proposed, which can better describe stability feature of tempered fractional systems. Third, tempered fractional chaotic systems have more alterable dynamical behaviors than fractional ones. It may be more useful in secure communication and control processing. Finally, an auxiliary system approach is used to consider the generalized synchronization for fractional complex networks. This is another difference between the fractional complex network synchronization in this paper and those of previous papers.

The rest of this paper is organized as follows. Some necessary preliminaries are given in Section 2. Generalized synchronization is discussed in Section 3. Simulation results are given in Section 4. Finally, the paper is concluded in Section 5 .

\section{Properties of the Tempered Fractional Derivative}

Some definitions and properties are introduced in this section.

Definition 1 (see [9]). For $n-1 \leq \alpha<n, n \in \mathbb{N}$, and $\lambda \geq 0$, the tempered fractional Caputo derivative is defined as

$$
{ }_{C} D_{t_{0}, t}^{\alpha, \lambda} x(t)=\frac{e^{-\lambda t}}{\Gamma(n-\alpha)} \int_{t_{0}}^{t} \frac{1}{(t-s)^{\alpha-n+1}} \frac{\mathrm{d}^{n}\left(e^{\lambda s} x(s)\right)}{\mathrm{d} s^{n}} \mathrm{~d} s
$$

where $\Gamma(\cdot)$ denotes the Gamma function defined by

$$
\Gamma(z)=\int_{0}^{\infty} e^{-t} t^{z-1} \mathrm{~d} t .
$$

Remark 1. If the tempered parameter $\lambda=0$, the tempered fractional Caputo derivative (2) reduces to the corresponding fractional Caputo derivative.

Consider the tempered Caputo fractional nonautonomous system

$$
{ }_{C} D_{0, t}^{\alpha, \lambda} x(t)=f(t, x),
$$

with initial condition $x(0)$, where $\alpha \in(0,1), \lambda \geq 0$, $f:[0,+\infty)$ is piecewise continuous in $t$ and locally Lipschitz in $x$, and $\Omega \subset \mathbb{R}^{n}$ is a domain that contains the origin $x=0$.

Definition 2. The constant $x_{0}$ is an equilibrium point of the tempered Caputo fractional dynamic system (4) if and only if

$$
{ }_{C} D_{0, t}^{\alpha, \lambda} x_{0}=f\left(t, x_{0}\right)
$$

For convenience, assume the equilibrium point in all definitions and theorems is $x_{0}=0$. Because any equilibrium point can be shifted to the origin via a change of variables, there is no loss of generality in doing so. Suppose the equilibrium point for (5) is $\bar{x} \neq 0$, and consider the change of variable $y=x-\bar{x}$. The tempered Caputo fractional derivative of $y$ is given by

$$
\begin{aligned}
{ }_{C} D_{0, t}^{\alpha, \lambda} y & ={ }_{C} D_{0, t}^{\alpha, \lambda}(x-\bar{x})=f(t, x)-\bar{x} e^{-\lambda t}{ }_{C} D_{0, t}^{\alpha, \lambda} e^{\lambda t} \\
& =f(t, y+\bar{x})-\bar{x} e^{-\lambda t}{ }_{C} D_{0, t}^{\alpha, \lambda} e^{\lambda t} \\
& =\bar{g}(t, y),
\end{aligned}
$$

where $\bar{g}(t, 0)=0$, and in the new variable $y$, the system has equilibrium at the origin.

Definition 3. The solution of (4) is said to be tempered Mittag-Leffler stable if

$$
\|x(t)\| \leq\left[m(x(0)) e^{-\lambda t} E_{\alpha}\left(-k t^{\alpha}\right)\right]^{b},
$$




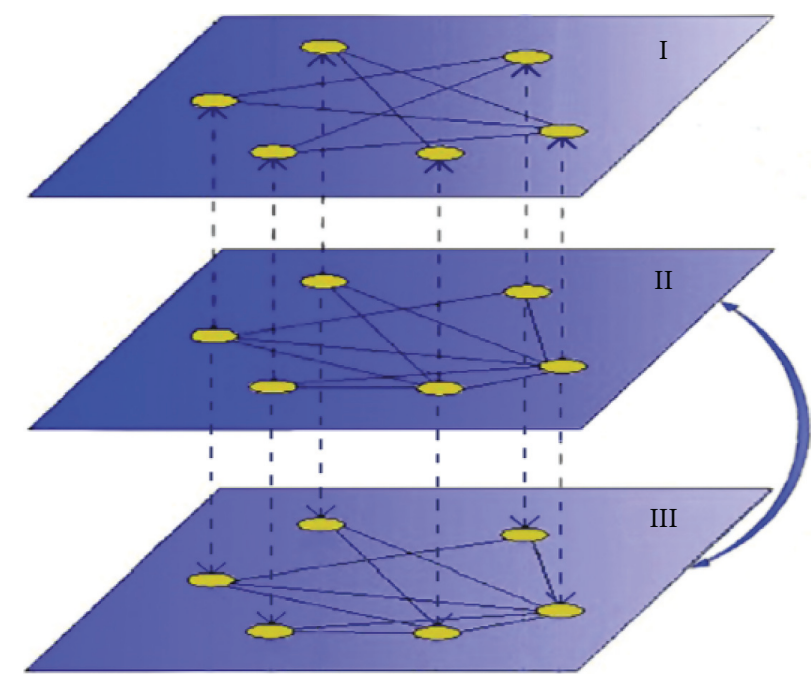

FIgURE 1: A schematic diagram [28] of generalized synchronization via the auxiliary system approach.

where $t \geq 0, \lambda \geq 0, \alpha \in(0,1), b>0, k \geq 0, m(0)=0, m(x) \geq 0$, and $m(x)$ is locally Lipschitz on $x \in \mathbb{B} \subset \mathbb{R}^{n}$ with Lipschitz constant $m_{0}$.

Remark 2. If $\lambda=0$, tempered Mittag-Leffler stability reduces to Mittag-Leffler stability.

Remark 3. Mittag-Leffler stability and tempered MittagLeffler stability imply asymptotic stability, that is, $\lim _{t \rightarrow \infty}\|x(t)\|=0$.

Definition 4 (see [30]). The Mittag-Leffler function is defined as

$$
E_{\alpha}(z)=\sum_{k=1}^{\infty} \frac{z^{k}}{\Gamma(k \alpha+1)}
$$

where $\alpha>0$. The Mittag-Leffler function with two parameters is defined as

$$
E_{\alpha, \beta}(z)=\sum_{k=1}^{\infty} \frac{z^{k}}{\Gamma(k \alpha+\beta)},
$$

where $\alpha>0$ and $\beta>0$.

For $\beta=1$, we have $E_{\alpha, 1}(z)=E_{\alpha}(z)$ and $E_{1,1}(z)=e^{z}$. The Laplace transform of the Mittag-Leffler function in two parameters is

$$
\begin{array}{r}
\mathscr{L}\left\{t^{\beta-1} E_{\alpha, \beta}\left(-\lambda t^{\alpha}\right)\right\}=\frac{s^{\alpha-\beta}}{s^{\alpha}+\lambda}, \\
\mathscr{R}(s)>|\lambda|^{1 / \alpha},
\end{array}
$$

where $t$ and $s$ are variables in the time domain and Laplace domain, respectively. $\mathscr{R}(s)$ is the real part of $s, \lambda \in \mathbb{R}$, and $\mathscr{L}\{\cdot\}$ is the Laplace transform. below.

To obtain the main results, several lemmas are given
Lemma 1 (see [31]). Assume that $Q=\left(q_{i j}\right)_{N \times N}$ is symmetric. Let $M^{*}=\operatorname{diag}\{\underbrace{m_{1}^{*}, m_{2}^{*}, \ldots, m_{l}^{*}}_{l}, \underbrace{0, \ldots, 0}_{N-l}\}, 1 \leq l \leq N$, and $m_{i}^{*}>0 \quad(i=1,2, \ldots, l), \quad Q-M^{*}=\left(\begin{array}{cc}E-\widetilde{M}^{*} & S \\ S^{T} & Q_{l}\end{array}\right), \quad \tilde{M}^{*}=$ $\operatorname{diag}\left(m_{1}^{*}, \ldots, m_{l}^{*}\right)$, and $m^{*}=\min _{1 \leq i \leq l}\left\{m_{i}^{*}\right\} . Q_{l}$ is a block matrix of $Q$ obtained by removing its first $l(1 \leq l \leq N)$ rowcolumn pairs, and $E$ and $S$ are matrices with appropriate dimensions. When $m^{*}>\lambda_{\max }\left(E-S Q_{l}^{-1} S^{T}\right), Q-M^{*}<0$ is equivalent to $Q_{l}<0$.

Lemma 2 (see [32]). The Laplace transform of tempered fractional Caputo derivative (2) is given as

$$
\begin{aligned}
\mathscr{L}\left\{{ }_{C} D_{0, t}^{\alpha, \lambda} x(t)\right\}= & (s+\lambda)^{\alpha} X(s) \\
& -\left.\sum_{k=0}^{n-1}(s+\lambda)^{\alpha-k-1}\left[\frac{\mathrm{d}^{k}}{\mathrm{~d} t^{k}}\left(e^{\lambda t} x(t)\right)\right]\right|_{t=0},
\end{aligned}
$$

where $X(s)=\mathscr{L}\{x(t)\}$ denotes the Laplace transform of $x(t)$.

We can obtain the following theorems for the tempered fractional derivative.

Theorem 1. Assume $x(t) \in \mathbb{R}$ is a continuous and differentiable function; then, for any time instant $t \geq 0$,

$$
{ }_{C} D_{0, t}^{\alpha, \lambda} x^{2}(t) \leq 2 x(t){ }_{C} D_{0, t}^{\alpha, \lambda} x(t),
$$

where $0<\alpha<1$ and $\lambda \geq 0$.

Proof. Let $y(s)=e^{\lambda s}(x(t)-x(s))^{2}, s \in[0, t]$. According to Definition 1 , for any $t \geq 0$, we have 


$$
\begin{aligned}
& { }_{C} D_{0, t}^{\alpha, \lambda} x^{2}(t) \\
& =\frac{e^{-\lambda t}}{\Gamma(1-\alpha)} \int_{0}^{t} \frac{1}{(t-s)^{\alpha}}\left[\lambda e^{\lambda s} x^{2}(s)+2 e^{\lambda s} x(s) \dot{x}(s)\right] \mathrm{d} s \\
& =\frac{e^{-\lambda t}}{\Gamma(1-\alpha)} \int_{0}^{t} \frac{e^{\lambda s}}{(t-s)^{\alpha}}\left[\lambda x^{2}(s)+2 x(s) \dot{x}(s)-2 \lambda x(t) x(s)\right. \\
& -2 x(t) \dot{x}(s)+2 \lambda x(t) x(s)+2 x(t) \dot{x}(s)] \mathrm{d} s \\
& =\frac{e^{-\lambda t}}{\Gamma(1-\alpha)} \int_{0}^{t} \frac{e^{\lambda s}}{(t-s)^{\alpha}}\left[\lambda x^{2}(s)+2 x(s) \dot{x}(s)-2 \lambda x(t) x(s)\right. \\
& -2 x(t) \dot{x}(s)] \mathrm{d} s+2 x(t)_{C} D_{0, t}^{\alpha, \lambda} x(t) \\
& \leq \frac{e^{-\lambda t}}{\Gamma(1-\alpha)} \int_{0}^{t} \frac{e^{\lambda s}}{(t-s)^{\alpha}}\left[\lambda^{x}(s)-2 \lambda x(t) x(s)+\lambda x^{2}(t)\right. \\
& +2 x(s) \dot{x}(s)-2 x(t) \dot{x}(s)] \mathrm{d} s+2 x(t)_{C} D_{0, t}^{\alpha, \lambda} x(t) \\
& =\frac{e^{-\lambda t}}{\Gamma(1-\alpha)} \int_{0}^{t} \frac{e^{\lambda s}}{(t-s)^{\alpha}}\left[\lambda(x(t)-x(s))^{2}+2 x(s) \dot{x}(s)\right. \\
& -2 x(t) \dot{x}(s)] \mathrm{d} s+2 x(t)_{C} D_{0, t}^{\alpha, \lambda} x(t) \\
& =\frac{e^{-\lambda t}}{\Gamma(1-\alpha)} \int_{0}^{t} \frac{1}{(t-s)^{\alpha}} \dot{y}(s) d s+2 x(t)_{C} D_{0, t}^{\alpha, \lambda} x(t) \\
& =\frac{e^{-\lambda t}}{\Gamma(1-\alpha)}\left[\left.\frac{y(s)}{(t-s)^{\alpha}}\right|_{0} ^{t}-\alpha \int_{0}^{t} \frac{y(s)}{(t-s)^{\alpha+1}} \mathrm{~d} s\right]+2 x(t)_{C} D_{0, t}^{\alpha, \lambda} x(t) \\
& \leq \frac{e^{-\lambda t}}{\Gamma(1-\alpha)} \lim _{s \longrightarrow t} \frac{y(s)}{(t-s)^{\alpha}}+2 x(t)_{C} D_{0, t}^{\alpha, \lambda} x(t) .
\end{aligned}
$$

Because the function $y(s)$ is differentiable, by L'Hôpital's rule, we obtain

$$
\begin{aligned}
& \frac{e^{-\lambda t}}{\Gamma(1-\alpha)} \lim _{s \longrightarrow t} \frac{y(s)}{(t-s)^{\alpha}} \\
& =-\frac{e^{-\lambda t}}{\Gamma(1-\alpha)} \lim _{s \longrightarrow t}\left[\frac{\lambda e^{\lambda s}(x(t)-x(s))^{2}}{\alpha(t-s)^{\alpha-1}}\right. \\
& \left.\quad+\frac{2 e^{\lambda s} \dot{x}(s)(x(s)-x(t))}{\alpha(t-s)^{\alpha-1}}\right] \\
& =-\frac{e^{-\lambda t}}{\alpha \Gamma(1-\alpha)} \lim _{s \longrightarrow t} e^{\lambda s}\left[\lambda(x(t)-x(s))^{2}\right. \\
& \quad+2 \dot{x}(s)(x(s)-x(t))](t-s)^{1-\alpha} \\
& =0 .
\end{aligned}
$$

Combining (13) and (14), we obtain

$$
{ }_{C} D_{0, t}^{\alpha, \lambda} x^{2}(t) \leq 2 x(t)_{C} D_{0, t}^{\alpha, \lambda} x(t) \text {. }
$$

The proof is completed.

Theorem 2. Let $x(t) \in \mathbb{R}^{n}$ be a differentiable vector function. Then, for any time instant $t \geq 0$, the following inequality holds:

$$
{ }_{C} D_{0, t}^{\alpha, \lambda}\left(x^{T}(t) P x(t)\right) \leq 2 x^{T}(t) P_{C} D_{0, t}^{\alpha, \lambda} x(t)
$$

where $0<\alpha<1, \lambda \geq 0$, and $P$ is a symmetric and positive definite matrix.

Proof. Since $P$ is a symmetric and positive definite matrix, there exists an orthogonal matrix $B \in \mathbb{R}^{n \times n}$ and a diagonal matrix $\Lambda \in \mathbb{R}^{n \times n}$, such that

$$
P=B \Lambda B^{T},
$$

where $\Lambda=\operatorname{diag}\left\{\lambda_{11}, \lambda_{22}, \ldots, \lambda_{n n}\right\}, \quad \lambda_{i i}>0(i=1,2, \ldots, n)$. Then,

$$
\begin{aligned}
x^{T}(t) P x(t) & =x^{T}(t) B \Lambda B^{T} x(t) \\
& =\left(B^{T} x(t)\right)^{T} \Lambda\left(B^{T} x(t)\right) .
\end{aligned}
$$

Define an auxiliary variable $y(t)=B^{T} x(t)$. One can then write

$$
x^{T}(t) P x(t)=y^{T}(t) \Lambda y(t)=\sum_{i=1}^{n} \lambda_{i i} y_{i}^{2}(t) .
$$

Using Theorem 1, one has

$$
\begin{aligned}
{ }_{C} D_{0, t}^{\alpha, \lambda}\left(x^{T}(t) P x(t)\right) & ={ }_{C} D_{0, t}^{\alpha, \lambda}\left(\sum_{i=1}^{n} \lambda_{i i} y_{i}^{2}(t)\right) \\
& =\sum_{i=1}^{n}{ }_{C} D_{0, t}^{\alpha, \lambda}\left(\lambda_{i i} y_{i}^{2}(t)\right) \\
& \leq 2 \sum_{i=1}^{n} \lambda_{i i} y_{i}(t)_{C} D_{0, t}^{\alpha, \lambda} y_{i}(t) \\
& =2 y^{T}(t) \Lambda_{C} D_{0, t}^{\alpha, \lambda} y(t) \\
& =2\left(B^{T} x(t)\right)^{T} \Lambda_{C} D_{0, t}^{\alpha, \lambda}\left(B^{T} x(t)\right) \\
& =2 x^{T}(t) P_{C} D_{0, t}^{\alpha, \lambda} x(t),
\end{aligned}
$$

which completes the proof.

Theorem 3. Let $x(t) \in \mathbb{R}^{n}$ be a differentiable vector function. If a continuous function $V:[0,+\infty)$ satisfies

$$
{ }_{C} D_{0, t}^{\alpha, \lambda} V(t, x(t)) \leq-\theta V(t, x(t)),
$$

then

$$
V(t, x(t)) \leq V(0, x(0)) e^{-\lambda t} E_{\alpha}\left(-\theta t^{\alpha}\right)
$$

where $0<\alpha<1, \lambda \geq 0$, and $\theta$ is a positive constant.

Proof. From inequality (21), there exists a nonnegative function $M(t)$ satisfying 


$$
{ }_{C} D_{0, t}^{\alpha, \lambda} V(t, x(t))+\theta V(t, x(t))+M(t)=0 .
$$

Taking the Laplace transform (11) of (23), we obtain

$$
(s+\lambda)^{\alpha} V(s)-(s+\lambda)^{\alpha-1} V(0, x(0))+\theta V(s)+M(s)=0,
$$

where $V(s)=\mathscr{L}\{V(t, x(t))\}$ and $M(s)=\mathscr{L}\{M(t)\}$. Therefore,

$$
V(s)=\frac{(s+\lambda)^{\alpha-1} V(0, x(0))-M(s)}{(s+\lambda)^{\alpha}+\theta} .
$$

Taking the inverse Laplace transform of (25), we have

$$
\begin{aligned}
V(t, x(t))= & V(0, x(0)) e^{-\lambda t} E_{\alpha}\left(-\theta t^{\alpha}\right) \\
& -M(t) *\left[t^{\alpha-1} e^{-\lambda t} E_{\alpha, \alpha}\left(-\theta t^{\alpha}\right)\right],
\end{aligned}
$$

where * means convolution. Because both $M(t)$ and $t^{\alpha-1} e^{-\lambda t} E_{\alpha, \alpha}\left(-\theta t^{\alpha}\right)$ are nonnegative functions, we can obtain

$$
V(t, x(t)) \leq V(0, x(0)) e^{-\lambda t} E_{\alpha}\left(-\theta t^{\alpha}\right) .
$$

This completes the proof.

Actually, an appropriate function $V(t, x(t))$ that satisfies the inequality (21) is difficult to find. Therefore, we present a second Lyapunov method to weaken the conditions in Theorem 3.

Theorem 4. Let domain $\mathbb{D} \subset \mathbb{R}^{n}$ and system (4) all contain equilibrium point $x=0$, and assume $V(t, x(t)):[0,+\infty)$ satisfies

$$
\begin{gathered}
\alpha_{1}\|x(t)\|^{a} \leq V(t, x(t)) \leq \alpha_{2} x(t)^{a b}, \\
{ }_{C} D_{0, t}^{\alpha, \lambda} V\left(t^{+}, x\left(t^{+}\right)\right) \leq-\alpha_{3}\|x(t)\|^{\mathrm{ab}}
\end{gathered}
$$$$
\text { (holding almost everywhere), }
$$

and that $V(t, x(t))$ is locally Lipschitz with respect to $x, V(t, x(t))$ is piecewise continuous, $\lim _{\tau \longrightarrow t^{+}} \dot{V}(\tau, x(\tau))$ exists, and $V\left(t^{+}, x\left(t^{+}\right)\right) \triangleq \lim _{\tau \longrightarrow t^{+}} V(\tau, x(\tau)), \quad$ where $t \geq 0, x \in \mathbb{D}, \alpha \in$ $(0,1), \lambda \geq 0, \alpha_{1}, \alpha_{2}, \alpha_{3}, a$, and $b$ are given positive constants. Then, $x=0$ is tempered Mittag-Leffler stable. If the assumptions hold globally on $\mathbb{R}^{n}$, then $x=0$ is globally tempered Mittag-Leffler stable.

Proof. From equations (28) and (29), we obtain

$$
{ }_{C} D_{0, t}^{\alpha, \lambda} V\left(t^{+}, x\left(t^{+}\right)\right) \leq-\frac{\alpha_{3}}{\alpha_{2}} V(t, x(t))
$$

(holding almost everywhere).

There exists a nonnegative function $M(t)$ satisfying

$$
\begin{aligned}
{ }_{C} D_{0, t}^{\alpha, \lambda} V\left(t^{+}, x\left(t^{+}\right)\right)+M(t) \\
\quad=-\frac{\alpha_{3}}{\alpha_{2}} V(t, x(t))_{C} D_{0, t}^{\alpha, \lambda} V\left(t^{+}, x\left(t^{+}\right)\right) \\
\leq-\frac{\alpha_{3}}{\alpha_{2}} V(t, x(t))
\end{aligned}
$$

(holding almost everywhere).

Taking the Laplace transform of (31), we have

$$
(s+\lambda)^{\alpha} V^{+}(s)-(s+\lambda)^{\alpha-1} V\left(0^{+}\right)+M(s)=-\frac{\alpha_{3}}{\alpha_{2}} V(s),
$$

where $V\left(0^{+}\right)=\lim _{\tau \longrightarrow 0^{+}} V(\tau, x(\tau)) \geq 0 \quad$ and $\quad V^{+}(s)=\mathscr{L}$ $\left\{V\left(t^{+}, x\left(t^{+}\right)\right)\right\}$. Due to continuity of function $V(t, x(t))$ and (32), we obtain $V\left(t^{+}, x\left(t^{+}\right)\right)=V(t, x(t)), V^{+}(s)=V(s)$ and

$$
V(s)=\frac{V(0)(s+\lambda)^{\alpha-1}-M(s)}{(s+\lambda)^{\alpha}+\left(\alpha_{3} / \alpha_{2}\right)} .
$$

Applying the inverse Laplace transform, the unique solution of (33) is

$$
\begin{aligned}
V(t)= & V(0) e^{-\lambda t} E_{\alpha}\left(-\frac{\alpha_{3}}{\alpha_{2}} t^{\alpha}\right) \\
& -M(t) *\left[e^{-\lambda t} t^{\alpha-1} E_{\alpha, \alpha}\left(-\frac{\alpha_{3}}{\alpha_{2}} t^{\alpha}\right)\right] .
\end{aligned}
$$

Because $t^{\alpha-1}, e^{-\lambda t}$, and $E_{\alpha, \alpha}\left(-\left(\alpha_{3} / \alpha_{2}\right) t^{\alpha}\right)$ are nonnegative functions, we obtain

$$
V(t) \leq V(0) e^{-\lambda t} E_{\alpha}\left(-\frac{\alpha_{3}}{\alpha_{2}} t^{\alpha}\right)
$$

Substituting (35) into (28) gives

$$
\|x(t)\| \leq\left[\frac{V(0)}{\alpha_{1}} e^{-\lambda t} E_{\alpha}\left(-\frac{\alpha_{3}}{\alpha_{2}} t^{\alpha}\right)\right]^{1 / a} .
$$

Let $m=\left(V(0) / \alpha_{1}\right) \geq 0$. It follows from (26) that

$$
x(t) \leq\left[\mathrm{me}^{-\lambda t} E_{\alpha}\left(-\frac{\alpha_{3}}{\alpha_{2}} t^{\alpha}\right)\right]^{1 / a},
$$

where $x(0)=0$ if and only if $\left(V(0) / \alpha_{1}\right)=0$.

Since $V(t, x)$ about $x$ satisfies the local Lipschitz condition, we can obtain $(V(0, x(0))) / \alpha_{1}$ near $x(0)$ which satisfies the local Lipschitz condition. This result also means system (4) is tempered Mittag-Leffler stable, which completes the proof.

Remark 4. In Theorems $1-4$, if $\lambda=0$, one has some properties about fractional derivative and fractional system, which have been discussed [33-36]. So, the results are more general.

Remark 5. It should be noted that we pay special attention to the tempered Mittag-Leffler stability for the following reasons. On one hand, the tempered Mittag-Leffler stability is essential in the synchronization analysis of tempered fractional complex networks. On the other hand, the tempered Mittag-Leffler stability is an important character when evaluating the tempered fractional system.

\section{Generalized Synchronization Criteria for Two-Layer Tempered Fractional Networks via Pinning Control}

In this section, a pinning control method for achieving the generalized synchronization between two-layer tempered fractional complex networks is presented. 
Consider the following tempered fractional complex network composed of $N$ unidirectionally coupled nodes that is described by

$$
\begin{aligned}
{ }_{C} D_{0, t}^{\alpha_{1}, \lambda_{1}} x_{i}(t)= & H x_{i}(t)+f\left(x_{i}(t)\right) \\
& +\varepsilon_{1} \sum_{j=1}^{N} a_{i j} P x_{j}(t), \quad i=1,2, \ldots, N,
\end{aligned}
$$

where $0<\alpha_{1}<1, \lambda_{1} \geq 0$, and $x_{i}(t)=\left(x_{i 1}(t), x_{i 2}(t), \ldots, x_{i n}\right.$ $(t))^{T} \in \mathbb{R}^{n}$ is the state vector of the $i$-th node. $H x_{i}(t)$ and $f\left(x_{i}\right.$ $(t))=\left(f_{1}\left(x_{i}(t)\right), f_{2}\left(x_{i}(t)\right), \ldots, f_{n}\left(x_{i}(t)\right)\right)^{T}: \mathbb{R}^{n} \longrightarrow \mathbb{R}^{n}$ are the linear and nonlinear parts of the isolated $i$-th node, respectively. $\varepsilon_{1}$ is the coupling strength. $P \in \mathbb{R}^{n \times n}$ is the positive definite diagonal inner-coupling matrix, and $A=\left(a_{i j}\right)_{N \times N}$ is the symmetric coupling configuration matrix, in which $a_{i j}$ is defined as follows: if there is a connection from node $j$ to node $i(i \neq j), a_{i j}=1$; otherwise, $a_{i j}=0$. The diagonal elements of matrix $A$ are defined as $a_{i i}=-\sum_{j=1, j \neq i}^{N} a_{i j}, i=1,2, \ldots, N$.

Equation (38) is regarded as the drive network, and the response network with $N$ coupling nodes is chosen as follows:

$$
\begin{aligned}
{ }_{C} D_{0, t}^{\alpha_{2}, \lambda_{2}} y_{i}(t)= & M y_{i}(t)+g\left(y_{i}(t)\right) \\
& +\varepsilon_{2} \sum_{j=1}^{N} b_{i j} Q y_{j}(t), \quad i=1,2, \ldots, N,
\end{aligned}
$$

where $y_{i}(t)=\left(y_{i 1}(t), y_{i 2}(t), \ldots, y_{i n}(t)\right)^{T} \in \mathbb{R}^{n}$ is the state vector. $M y_{i}(t)$ and $g\left(y_{i}(t)\right)$ are the linear and nonlinear parts of the isolated $i$-th node, respectively. $\varepsilon_{2}$ is the coupling strength. $Q$ and $B=\left(b_{i j}\right)_{N \times N}$ have the same meanings as those of $P$ and $A$ in network (38), respectively.

Without loss of generality, the first $l$ nodes are selected as the pinned nodes in the complex network (39). Therefore, the pinned network with pinning controllers $u_{i}\left(x_{i}, y_{i}\right)$, where $i=1,2, \ldots, l$, is described as follows:

$$
\begin{cases}{ }_{C} D_{0, t}^{\alpha_{2}, \lambda_{2}} y_{i}(t)=M y_{i}(t)+g\left(y_{i}(t)\right) & \\ \quad+\varepsilon_{2} \sum_{j=1}^{N} b_{i j} Q y_{j}(t)+u_{i}\left(x_{i}, y_{i}\right), & i=1,2, \ldots, l, \\ { }_{C} D_{0, t}^{\alpha_{2}, \lambda_{2}} y_{i}(t)=M y_{i}(t)+g\left(y_{i}(t)\right) & \\ +\varepsilon_{2} \sum_{j=1}^{N} b_{i j} Q y_{j}(t), & i=l+1, l+2, \ldots, N .\end{cases}
$$

Output signals from the drive network (39) are taken as input for the response network (40), so the latter will reach harmonious coexistence with the former.

To realize the generalized synchronization between the drive layer (39) and the response layer (40), an auxiliary network layer is selected as the following form:

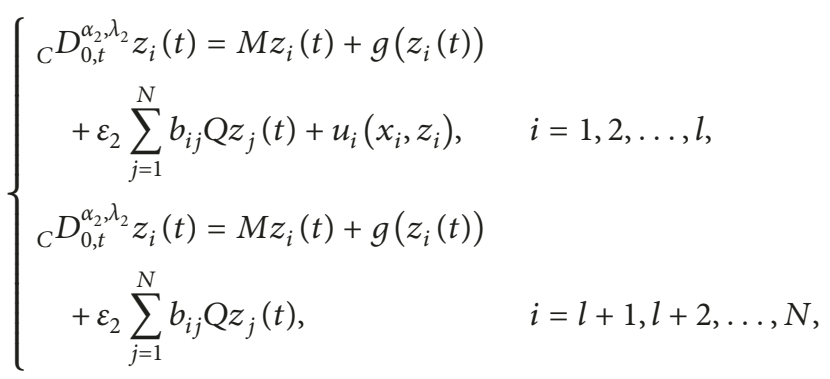

where $z_{i}$ is the state vector of the $i$-th node and $u_{i}\left(x_{i}, z_{i}\right)(i=$ $1,2, \ldots, l)$ are controllers of the same form as that of $u_{i}\left(x_{i}, y_{i}\right)$.

According to the auxiliary system approach, the drive layer (38) and response layer (40) are said to achieve generalized synchronization if the response network (40) and the auxiliary network (41) reach complete outer synchronization. If $\lim _{t \rightarrow \infty}\left\|y_{i}(t)-z_{i}(t)\right\|=0$ for any initial conditions $\quad y_{i}(0) \neq z_{i}(0)(i=1,2, \ldots, N)$, the generalized synchronization between the two-layer tempered fractional networks (38) and (40) is realized.

Hereafter, suppose that the nonlinear function $g(x(t))$ satisfies the Lipschitz condition. Then, there exists a nonnegative constant $L$ such that

$$
\|g(x(t))-g(y(t))\| \leq L\|x(t)-y(t)\|,
$$

for any time-varying vectors $x(t), y(t) \in \mathbb{R}^{n}$ with the norm $\|x\|=\sqrt{x^{T} x}$.

Define the synchronization error between (40) and (41) as $e_{i}(t)=z_{i}(t)-y_{i}(t)(i=1,2, \ldots, N)$. Then, the error dynamical network is described by

$$
\left\{\begin{array}{r}
{ }_{C} D_{0, t}^{\alpha_{2}, \lambda_{2}} e_{i}(t)=M e_{i}(t)+g\left(z_{i}\right)-g\left(y_{i}\right) \\
+\varepsilon_{2} \sum_{j=1}^{N} b_{i j} Q e_{j}(t)+u_{i}\left(x_{i}, z_{i}\right)-u_{i}\left(x_{i}, y_{i}\right) \\
i=1,2, \ldots, l, \\
{ }_{C} D_{0, t}^{\alpha_{2}, \lambda_{2}} e_{i}(t)=M e_{i}(t)+g\left(z_{i}\right)-g\left(y_{i}\right)+\varepsilon_{2} \sum_{j=1}^{N} b_{i j} Q e_{j}(t), \\
i=l+1, l+2, \ldots, N .
\end{array}\right.
$$

Theorem 5. The pinning controllers are chosen as

$$
\begin{aligned}
& u_{i}\left(x_{i}, z_{i}\right)=-k_{i}\left(z_{i}-x_{i}\right), \quad i=1,2, \ldots, l, \\
& u_{i}\left(x_{i}, y_{i}\right)=-k_{i}\left(y_{i}-x_{i}\right), \quad i=1,2, \ldots, l .
\end{aligned}
$$

Let $q=\|Q\|, K=\operatorname{diag}(k_{1}, k_{2}, \ldots, k_{l}, \underbrace{0, \ldots, 0}_{N-l}), I_{N}$ be an $N \times N$ identity matrix, $\widetilde{B}$ be a modified matrix of matrix $B$ by replacing the diagonal elements $b_{i i}$ with $\rho_{\min } b_{i i} / q$, and $k=\min _{1 \leq i \leq l}\left\{k_{i}\right\}$. If Lipschitz condition (42) and the condition

$$
\left(L+\lambda_{M}\right) I_{N}+\varepsilon_{2} q \widetilde{B}-K<0
$$

are satisfied, then the drive network (38) and the response network (40) can reach generalized synchronization with controllers (44) and (45). 
Specifically, for a suitable $k$, if there exists a natural number $1 \leq l<N$ such that the largest eigenvalue of $\widetilde{B}$ satisfies $L+\lambda_{M}+\varepsilon_{2} q \lambda_{\max }\left(\widetilde{B}_{l+1}\right)<0$, then the tempered fractional response network (40) asymptotically synchronizes to the drive network (38) with the pinning scheme (44) and (45).

Proof. According to (44) and (45), the error network (43) can be rewritten as

$$
\left\{\begin{aligned}
{ }_{C} D_{0, t}^{\alpha_{2}, \lambda_{2}} e_{i}(t)=M e_{i}(t)+g\left(z_{i}\right)-g\left(y_{i}\right) & \\
\quad+\varepsilon_{2} \sum_{j=1}^{N} b_{i j} Q e_{j}(t)-k_{i}\left(z_{i}-y_{i}\right), & i=1,2, \ldots, l, \\
{ }_{C} D_{0, t}^{\alpha_{2}, \lambda_{2}} e_{i}(t)=M e_{i}(t) & \\
& +g\left(z_{i}\right)-g\left(y_{i}\right)+\varepsilon_{2} \sum_{j=1}^{N} b_{i j} Q e_{j}(t), \quad i=l+1, l+2, \ldots, N .
\end{aligned}\right.
$$

Consider the Lyapunov function candidate

$$
V(t)=\frac{1}{2} \sum_{i=1}^{N} e_{i}^{T}(t) e_{i}(t)
$$

According to Theorem 2, its tempered fractional calculus along the trajectory of (47) is

$$
\begin{aligned}
&{ }_{C} D_{0, t}^{\alpha_{2}, \lambda_{2}} V(t) \leq \sum_{i=1}^{N} e_{i}^{T}(t)_{C} D_{0, t}^{\alpha_{2}, \lambda_{2}} e_{i}(t) \\
& \leq \sum_{i=1}^{N} e_{i}^{T}(t) M e_{i}(t)+\sum_{i=1}^{N} e_{i}^{T}(t)\left[g\left(z_{i}\right)-g\left(y_{i}\right)\right] \\
& \quad+\varepsilon_{2} \sum_{i=1}^{N} \sum_{j=1}^{N} b_{i j} e_{i}^{T}(t) Q e_{j}(t)-\sum_{i=1}^{l} k_{i} e_{i}^{T}(t) e_{i}(t) \\
& \leq \sum_{i=1}^{N} e_{i}^{T}(t)\left(\frac{M+M^{T}}{2}\right) e_{i}(t)+L \sum_{i=1}^{N} e_{i}^{T}(t) e_{i}(t) \\
& \quad+\varepsilon_{2} \sum_{i=1}^{N} \sum_{j=1, j \neq i}^{N} b_{i j} e_{i}^{T}(t) Q e_{j}(t) \\
& \quad+\varepsilon_{2} \sum_{i=1}^{N} b_{i i} e_{i}^{T}(t)\left(\frac{Q+Q^{T}}{2}\right) e_{i}(t)-\sum_{i=1}^{l} k_{i} e_{i}^{T}(t) e_{i}(t) \\
& \quad+\varepsilon_{2} q \sum_{i=1}^{N} \sum_{j=1, j \neq i}^{N} b_{i j}\left\|e_{i}(t)\right\| \cdot\left\|e_{j}(t)\right\| \\
& \quad+\varepsilon_{2} q \sum_{i=1}^{N} \sum_{j=1, j \neq i}^{N} b_{i j}\left\|e_{i}(t)\right\| \cdot\left\|e_{j}(t)\right\| \\
&\left.+\lambda_{M}\right) \sum_{i=1}^{N} e_{i}^{T}(t) e_{i}(t)-\sum_{i=1} k_{i} e_{i}^{T}(t) e_{i}(t)
\end{aligned}
$$

where $\lambda_{M}$ is the maximum eigenvalue of matrix $\left(M+M^{T}\right) / 2, \rho_{\min }$ is the minimum eigenvalue of matrix $\left(Q+Q^{T}\right) / 2$, and $e=\left(e_{1}, e_{2}, \ldots,\left\|e_{N}\right\|\right)^{T}$.

If the condition $H=\left(L+\lambda_{M}\right) I_{N}+\varepsilon_{2} q \widetilde{B}-K<0$ holds, we have $\eta=-\lambda_{\max }(H)>0$ and

$$
{ }_{C} D_{0, t}^{\alpha_{2}, \lambda_{2}} V(t) \leq-\eta e^{T} e=-\eta V(t) \text {. }
$$

According to Theorem 3, we have

$$
V(t, x(t)) \leq V(0, x(0)) e^{-\lambda_{2} t} E_{\alpha_{2}}\left(-\eta t^{\alpha_{2}}\right),
$$

that is, $\left\|e_{i}(t)\right\| \longrightarrow 0(i=1,2, \ldots, N)$ as $t \longrightarrow \infty$, which means that the generalized synchronization between the drive network (38) and the response network (40) is realized.

Specifically, let $k=\min _{1 \leq i \leq l}\left\{k_{i}\right\}$ and $\widetilde{B}_{l+1}$ be the reduced matrix of $\widetilde{B}$ obtained by removing the first $l$ row-column pairs. According to Lemma $1, H<0$ is equivalent to $(L+$ $\left.\lambda_{M}\right) I_{N-l}+c q \widetilde{B}_{l+1}<0$ for suitable $k$. Since $H_{l+1}=(L+$ $\left.\lambda_{M}\right) I_{N-l}+\varepsilon_{2} q \widetilde{B}_{l+1}$ is a real symmetric matrix, the largest eigenvalue $\lambda_{\max }\left(H_{l+1}\right)$ of $H_{l+1}$ is real and $\lambda_{\max }\left(H_{l+1}\right)=(L+$ $\left.\lambda_{M}\right)+\varepsilon_{2} q \lambda_{\max }\left(\widetilde{B}_{l+1}\right)$. Thus, if there exists a positive constant $1 \leq l \leq N$ such that $L+\lambda_{M}+\varepsilon_{2} q \lambda_{\max }\left(\widetilde{B}_{l+1}\right)<0$, one has $\left(L+\lambda_{M}\right) I_{N-l}+\varepsilon_{2} q \widetilde{B}_{l+1}<0$. Therefore, $H<0$, and we have obtained the desired results.

Remark 6. In Theorem 5, if $\lambda=0$, the results hold too, that is, the generalized synchronization in two-layer fractional complex networks via pinning control can be realized.

\section{Simulation Results}

In this section, a predictor-corrector algorithm and an example are provided to illustrate the effectiveness of the proposed method.

4.1. Predictor-Corrector Algorithm. The numerical calculation for tempered fractional systems is not as simple as that of an ordinary differential equation. Here, we use the generalized Adams-Bashforth-Moulton method [37, 38]. A brief introduction of this algorithm is given.

Consider the differential equation

$$
{ }_{C} D_{0, t}^{\alpha, \lambda} x(t)=f(t, x(t)), \quad 0<t<T,
$$

with initial conditions

$$
\left.\frac{\mathrm{d}^{k}}{\mathrm{~d} t^{k}}\left(e^{\lambda t} x(t)\right)\right|_{t=0}=c_{k}, \quad k=0,1, \ldots, \alpha-1,
$$

where $n \leq \alpha<n, n \in \mathbb{N}^{+}, \lambda>0$, and $c_{k}$ are arbitrary real numbers.

The tempered fractional equations (52) and (53) are equivalent to the following Volterra integral equation [32]:

$$
x(t)=x_{0}(t)+\frac{1}{\Gamma(\alpha)} \int_{0}^{t} e^{-\lambda(t-\tau)}(t-\tau)^{\alpha-1} f(\tau, x(\tau)) \mathrm{d} \tau,
$$

where $x_{0}(t)=\sum_{k=0}^{\alpha-1} c_{k}\left(\left(e^{-\lambda t} t^{k}\right) / k !\right)$. For uniform nodes $t_{n+1}=(n+1) h(n=0,1, \ldots, N)$, with $h=T / N$ being the 
step size of the numerical computation. Then, the corrector formula for (54) to compute $x_{n+1} \approx x\left(t_{n+1}\right)$ is

$$
x_{n+1}=\left\{\begin{array}{c}
x_{0}\left(t_{1}\right)+\frac{h^{\alpha}}{\Gamma(\alpha+2)}\left[f\left(t_{1}, x_{1}^{P}\right)\right. \\
\left.+\alpha e^{-\lambda h} f\left(0, x_{0}\right)\right], \quad \text { if } n=0, \\
x_{0}\left(t_{n+1}\right)+\frac{h^{\alpha}}{\Gamma(\alpha+2)}\left[\sum_{i=0}^{n} a_{i, n+1} f\left(t_{i}, x_{i}\right)\right. \\
\left.+f\left(t_{n+1}, x_{n+1}^{P}\right)\right],
\end{array}\right.
$$

where the predicted value $x_{n+1}^{P}$ is determined by

$$
\begin{aligned}
& x_{n+1}^{P}= \begin{cases}x_{0}\left(t_{1}\right)+\frac{h^{\alpha}}{\Gamma(\alpha+1)} e^{-\lambda h} f\left(0, x_{0}\right), & \text { if } n=0, \\
x_{0}\left(t_{n+1}\right)+\frac{h^{\alpha}}{\Gamma(\alpha+2)}\left[\sum_{i=0}^{n-1} a_{i, n+1} f\left(t_{i}, x_{i}\right)+e^{-\lambda h}\left(2^{\alpha+1}-1\right) \cdot f\left(t_{n}, x_{n}\right)\right], & \text { if } n \geq 1,\end{cases} \\
& a_{i, n+1}= \begin{cases}e^{-\lambda(n+1) h}\left[n^{\alpha+1}-(n+1)^{\alpha}(n-\alpha)\right], & \text { if } i=0, \\
e^{-\lambda(n+1-i) h}\left[(n-i)^{\alpha+1}-2(n+1-i)^{\alpha+1}+(n+2-i)^{\alpha+1}\right], & \text { if } 1 \leq i \leq n .\end{cases}
\end{aligned}
$$

The estimation error of this approximation is

$$
\max _{0 \leq n \leq N}\left|x\left(t_{n}\right)-x_{n}\right|= \begin{cases}O\left(h^{2}\right), & \text { if } \alpha \geq 0.5, \\ O\left(h^{1+2 \alpha}\right), & \text { if } 0<\alpha<0.5\end{cases}
$$

The numerical solution of a tempered fractional system can be determined by applying the above method.

4.2. Numerical Simulations. In this section, an example is provided to illustrate the effectiveness of the pinning strategies for the generalized synchronization in two-layer tempered fractional complex networks.

Now, we obtain a new tempered fractional chaotic system, that is, a generalization of the corresponding integer order system [39]. This chaotic system is adopted as the node dynamics in the layer of drive network (38), which is described as

$$
\left\{\begin{array}{l}
{ }_{C} D_{0, t}^{\alpha_{1}, \lambda_{1}} x_{i 1}=-x_{i 2} x_{i 3}+a_{1} x_{i 1}, \\
{ }_{C} D_{0, t}^{\alpha_{1}, \lambda_{1}} x_{i 2}=x_{i 1} x_{i 3}-b_{1} x_{i 2}, \\
{ }_{C} D_{0, t}^{\alpha_{1}, \lambda_{1}} x_{i 3}=\left(\frac{1}{3}\right) x_{i 1} x_{i 2}-c_{1} x_{i 3},
\end{array}\right.
$$

where $i=1,2, \ldots, 10, \alpha_{1}=0.99, \quad a_{1}=5, b_{1}=10, \quad$ and $c_{1}=3.8$. One can obtain the attractor of tempered fractional chaotic systems with different $\lambda$, as shown in Figure 2.

In the layer of the response network (39), tempered fractional Chen system [33] is chosen as the node dynamics:

$$
\left\{\begin{array}{l}
{ }_{C} D_{0, t}^{\alpha_{2}, \lambda_{2}} y_{i 1}=a_{2}\left(y_{i 2}-y_{i 1}\right), \\
{ }_{C} D_{0, t}^{\alpha_{2}, \lambda_{2}} y_{i 2}=\left(c_{2}-a_{2}\right) y_{i 1}-y_{i 1} y_{i 3}+c_{2} y_{i 2}, \\
{ }_{C} D_{0, t}^{\alpha_{2}, \lambda_{2}} y_{i 3}=y_{i 1} y_{i 2}-b_{2} y_{i 3},
\end{array}\right.
$$

where $i=1,2, \ldots, 10, \alpha_{2}=0.995, \quad a_{2}=35, b_{2}=3$, and $c_{2}=28$. One can obtain the attractor of the fractional chaotic Chen system, as shown in Figure 3. From Figures 2 and 3, we can see that the tempered fractional system has more abundant dynamical behavior than the corresponding fractional one. Additionally, in the layer of the response network (41), the dynamics of each node are in the same form as those in the response layer.

For convenience, let inner-coupling matrices $P=Q=I$. The coupling configuration matrices $A$ and $B$ are given as 


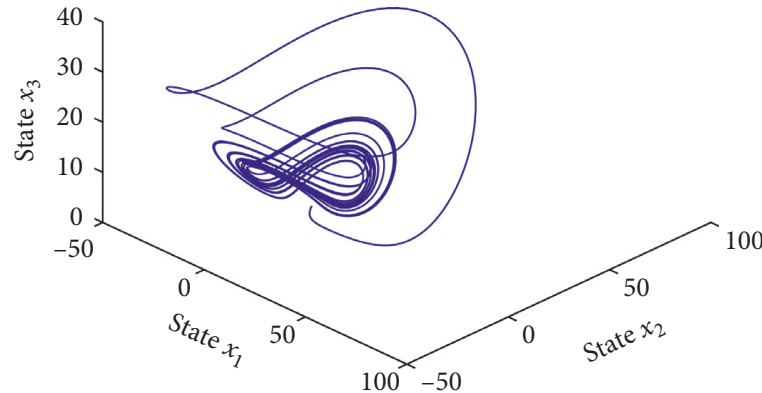

(a)

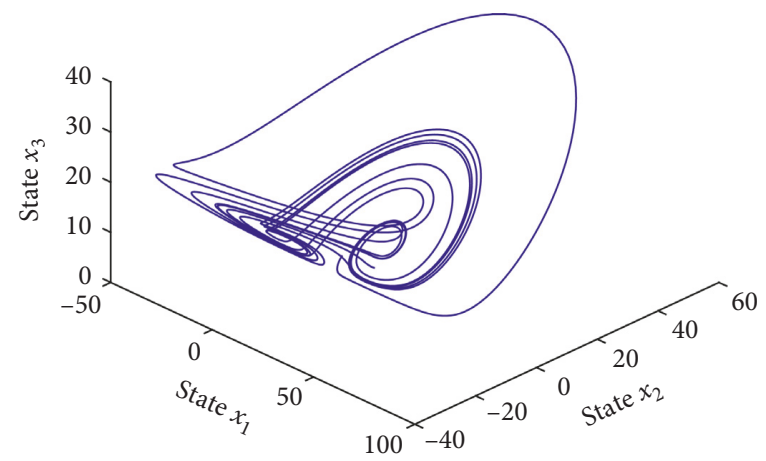

(c)

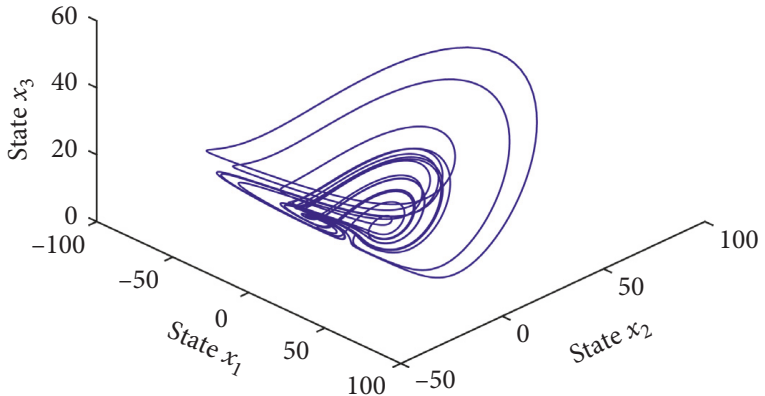

(b)

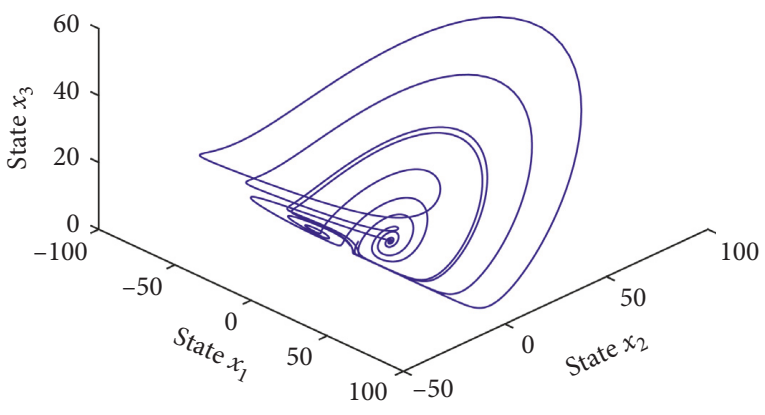

(d)

FIgURE 2: The attractor of the new tempered fractional system with different $\lambda_{1}$. (a) $\lambda_{1}=0$. (b) $\lambda_{1}=1$. (c) $\lambda_{1}=2$. (d) $\lambda_{1}=3$.

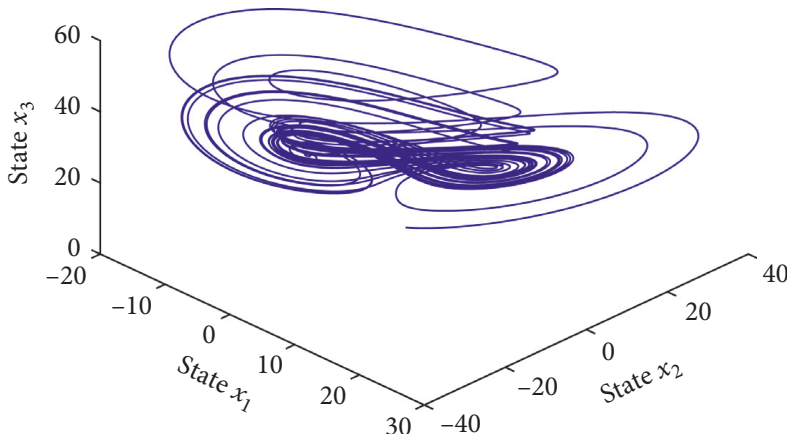

(a)

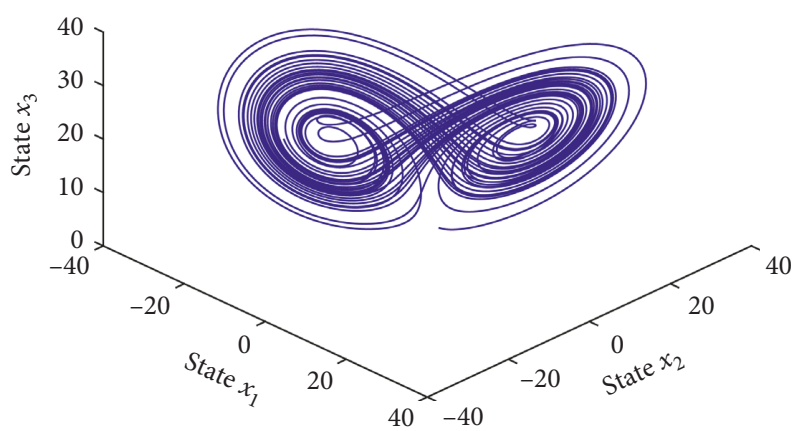

(c)

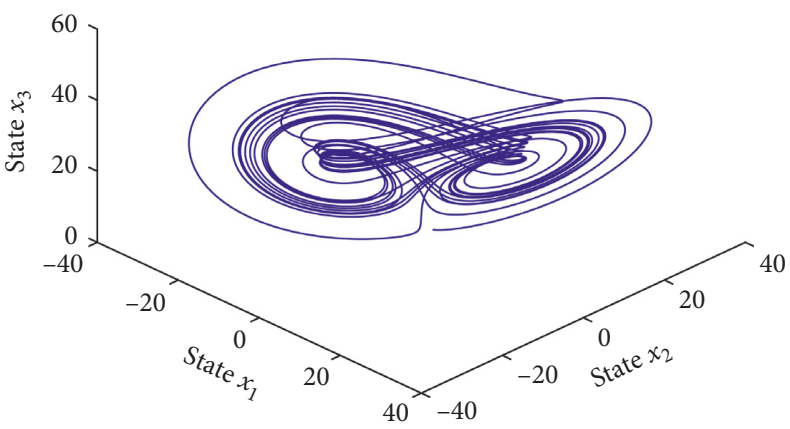

(b)

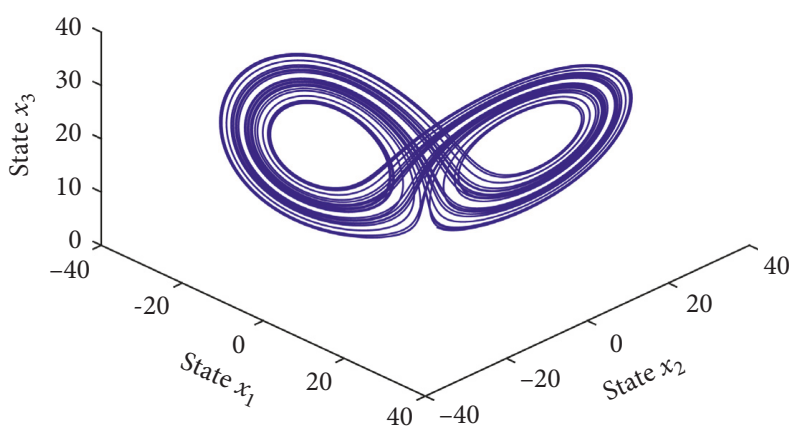

(d)

FIgURE 3: The attractor of the tempered fractional Chen system with different $\lambda_{2}$. (a) $\lambda_{2}=0$. (b) $\lambda_{2}=2$. (c) $\lambda_{2}=4$. (d) $\lambda_{2}=6$. 


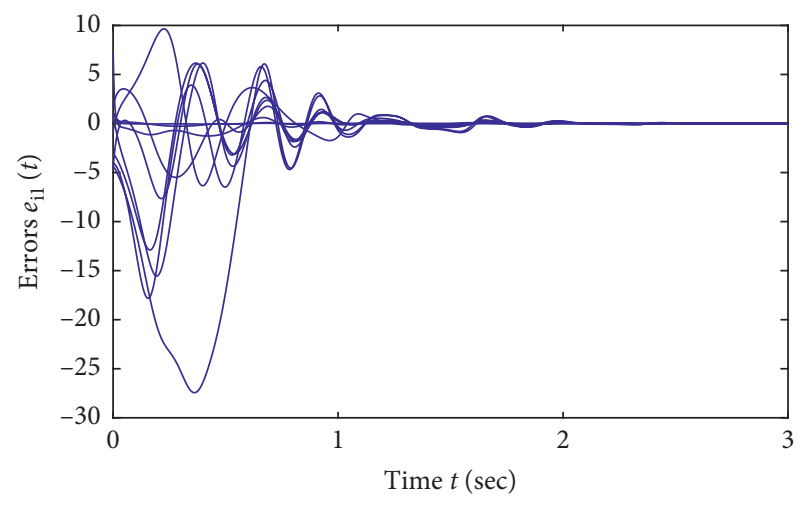

(a)

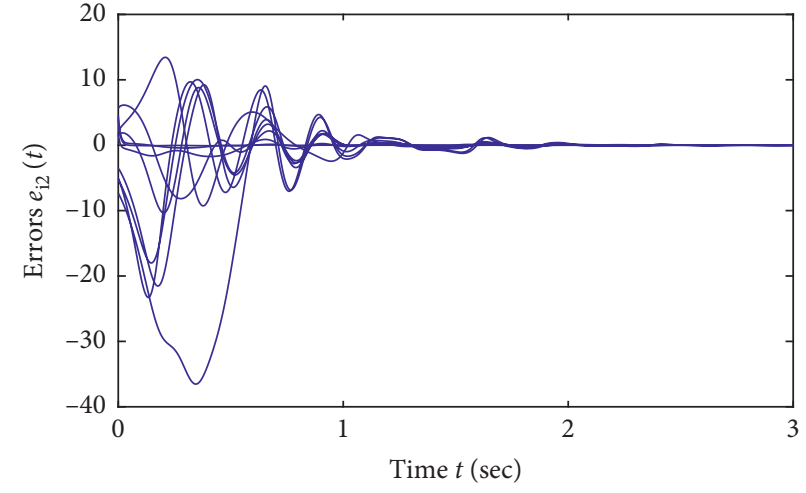

(b)

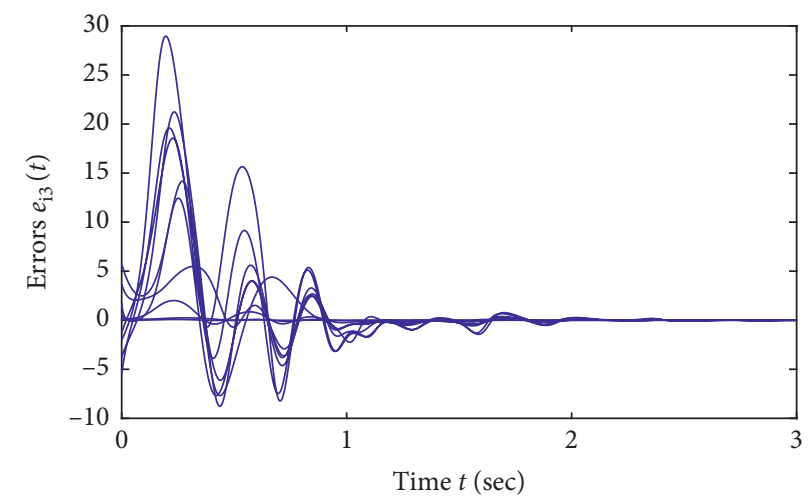

(c)

Figure 4: Time evolution of the error states $e_{i 1}, e_{i 2}$, and $e_{i 3}$ with $\lambda_{1}=1$ and $\lambda_{2}=2$. (a) $e_{i 1}(i=1,2, \ldots, 10)$. (b) $e_{i 2}(i=1,2, \ldots, 10)$. (c) $e_{i 3}(i=1,2, \ldots, 10)$.

$$
A=B=\left(\begin{array}{cccccccccc}
-2 & 1 & 0 & 1 & 0 & 0 & 0 & 0 & 0 & 0 \\
0 & -2 & 1 & 0 & 1 & 0 & 0 & 0 & 0 & 0 \\
1 & 0 & -3 & 0 & 0 & 1 & 1 & 0 & 0 & 0 \\
0 & 0 & 1 & -1 & 0 & 0 & 0 & 0 & 0 & 0 \\
0 & 1 & 0 & 1 & -3 & 0 & 0 & 0 & 1 & 0 \\
0 & 0 & 1 & 0 & 1 & -2 & 0 & 0 & 0 & 0 \\
0 & 0 & 0 & 0 & 0 & 1 & -3 & 1 & 0 & 1 \\
0 & 1 & 0 & 1 & 0 & 0 & 0 & -3 & 1 & 0 \\
1 & 0 & 1 & 0 & 0 & 0 & 1 & 0 & -4 & 1 \\
0 & 0 & 1 & 1 & 0 & 0 & 0 & 0 & 0 & -2
\end{array}\right) .
$$

Fractional Chen systems are bounded. Actually, $\left\|y_{i 1}\right\| \leq 60,\left\|y_{i 2}\right\|$ $\leq 40,\left\|y_{i 3}\right\| \leq 40,\left\|z_{i 1}\right\| \leq 60,\left\|z_{i 2}\right\| \leq 40,\left\|z_{i 3}\right\| \leq 40, i=1,2, \ldots, 10$, and $\left\|f\left(x_{i}\right)-f\left(y_{i}\right)\right\| \leq \sqrt{\left(-y_{i 1} y_{i 3}+z_{i 1} z_{i 3}\right)^{2}+\left(y_{i 1} y_{i 2}-z_{i 1} z_{i 2}\right)^{2}} \leq$ $84.85\left\|e_{i}\right\|$, that is, $L=84.85$. The out-degrees of nodes 2,3 , and 4 are larger than their in-degrees, so we select them as the pinned nodes [31]. After rearranging the network nodes, the new order is $4,3,2,1,6,10,5,7,8$, and 9 .

Let $k_{i}=120, \varepsilon_{1}=0.45$, and $\varepsilon_{2}=5$; we can obtain $\lambda_{\max }\left(\left(L+\lambda_{M}\right) I_{N}+\varepsilon_{2} q \widetilde{B}-K\right)=-26.2501<0$. From Theorem

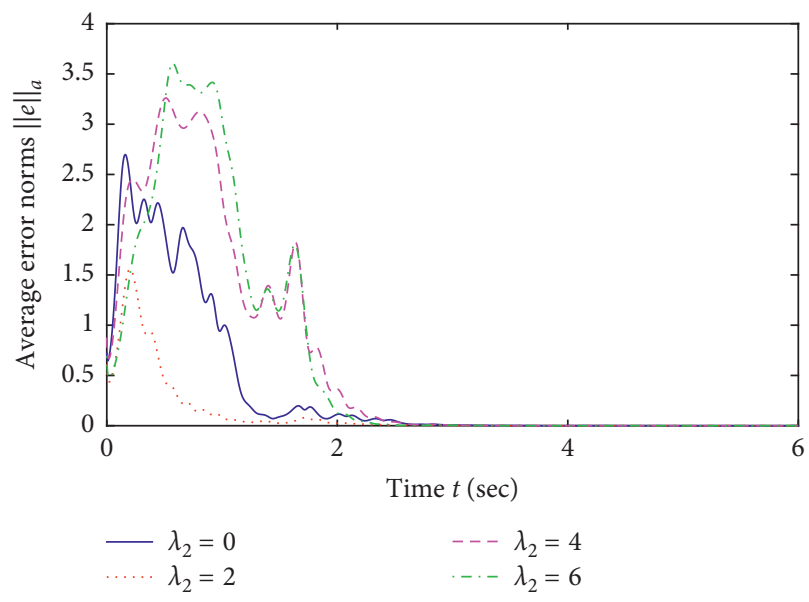

FIgURE 5: Time evolution of the average error norms $e_{a}$ with different tempered parameters $\lambda_{2}$.

5, the drive network (38) and the response network (40) can achieve generalized synchronization. The simulation results are shown in Figure 4, which illustrates the time waveforms of errors $e_{i 1}, e_{i 2}$, and $e_{i 3}(i=1,2, \ldots, 10)$.

The temper parameter $\lambda$ has a direct effect on the chaotic behavior of nonlinear dynamical systems. For convenience, we define the average error norm as 


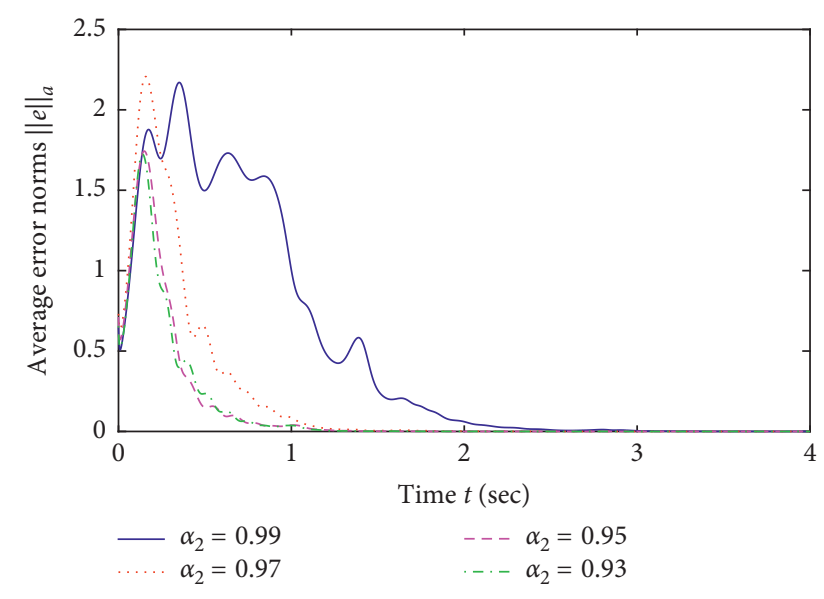

Figure 6: Time evolution of the average error norms $\|e\|_{a}$ with different fractional orders $\alpha_{2}$.

$$
\|e\|_{a}=\frac{1}{N} \sum_{i=1}^{N}\left\|e_{i}\right\|=\frac{1}{N} \sum_{i=1}^{N}\left\|y_{i}-z_{i}\right\| .
$$

We let the parameters depict as above, except $\lambda_{2}$. From Figure 5, the synchronization behaviors of fractional complex networks are realized with $\alpha_{2}=0.995$ and different tempered parameters $\lambda_{2}$. Furthermore, from Figure 6, fractional complex networks can reach synchronization for $\lambda_{2}=2$ and different fractional orders $\alpha_{2}$. The figures show that fractional complex networks (38) and (40) are synchronized, which demonstrate the effectiveness and robustness of the proposed method.

\section{Conclusion}

In this paper, the tempered fractional derivative is introduced to chaotic systems and complex networks. Synchronization of tempered fractional complex networks may be more useful in secure communication and control processing due to the addition of the tempered parameter. The pinning control scheme and auxiliary system approach are used to verify the existence of generalized synchronization in tempered fractional complex networks. Some properties of the tempered fractional derivative and tempered fractional system are discussed. Generalized synchronization criteria are established based on the proposed theory. The numerical results demonstrate the effectiveness of the proposed method. Furthermore, generalized synchronization can be achieved with different $\varepsilon_{2}$. Future work will study synchronization-based parameter identification [29] of tempered fractional delayed complex networks.

\section{Data Availability}

The data used to support the findings of this study are included within the article.

\section{Conflicts of Interest}

The authors declare that there are no conflicts of interest regarding the publication of this paper.

\section{Acknowledgments}

This work was supported by the National Natural Science Foundation of China (grant nos. 11872234 and 61966032), the Natural Science Foundation of Gansu Province, China (grant no. 17JR5RA284), and Gansu Provincial First-Class Discipline Program of Northwest Minzu University (no. 31920190047).

\section{References}

[1] A. Kiani-B, K. Fallahi, N. Pariz, and H. Leung, "A chaotic secure communication scheme using fractional chaotic systems based on an extended fractional Kalman filter," Communications in Nonlinear Science and Numerical Simulation, vol. 14 , no. 3, pp. 863-879, 2009.

[2] G. R. Chen and X. N. Dong, From Chaos to Order: Methodologies, Perspectives and Applications, World Scientific, Singapore, 1998.

[3] C. Yin, X. Huang, Y. Chen, S. Dadras, S.-m. Zhong, and Y. Cheng, "Fractional-order exponential switching technique to enhance sliding mode control," Applied Mathematical Modelling, vol. 44, pp. 705-726, 2017.

[4] C. Yin, X. Huang, S. Dadras et al., "Design of optimal lighting control strategy based on multi-variable fractional-order extremum seeking method," Information Sciences, vol. 465, pp. 38-60, 2018.

[5] F. Zhang, D. Lin, M. Xiao, and H. Li, "Dynamical behaviors of the chaotic brushless DC motors model," Complexity, vol. 21, no. 4, pp. 79-85, 2016.

[6] R. Metzler and J. Klafter, "The random walk's guide to anomalous diffusion: a fractional dynamics approach," Physics Reports, vol. 339, no. 1, pp. 1-77, 2000.

[7] Á. Cartea and D. del-Castillo-Negrete, "Fractional diffusion models of option prices in markets with jumps," Physica A: Statistical Mechanics and Its Applications, vol. 374, no. 2, pp. 749-763, 2007.

[8] A. Hanyga, "Wave propagation in media with singular memory," Mathematical and Computer Modelling, vol. 34, no. 12-13, pp. 1399-1421, 2001.

[9] Á. Cartea and D. del-Castillo-Negrete, "Fluid limit of the continuous-time random walk with general Lévy jump distribution functions," Physical Review E, vol. 76, Article ID 041105, 2007.

[10] M. M. Meerschaert, Y. Zhang, and B. Baeumer, "Tempered anomalous diffusion in heterogeneous systems," Geophysical Research Letters, vol. 35, no. 17, Article ID L17403, 2008.

[11] M. M. Meerschaert, F. Sabzikar, M. S. Phanikumar, and A. Zeleke, "Tempered fractional time series model for turbulence in geophysical flows," Journal of Statistical Mechanics: Theory and Experiment, vol. 2014, no. 9, Article ID P09023, 2014.

[12] F. Benedetto and G. Giunta, "A self-synchronizing method for asynchronous code acquisition in band-limited spread spectrum communications," IEEE Transactions on Communications, vol. 57, no. 8, pp. 2410-2419, 2009.

[13] E. Catsigeras, "Self-synchronization of networks with a strong kernel of integrate and fire excitatory neurons," WSEAS Transactions on Mathematics, vol. 12, no. 7, pp. 786-797, 2013.

[14] G. Velmurugan and R. Rakkiyappan, "Hybrid projective synchronization of fractional-order memristor-based neural networks with time delays," Nonlinear Dynamics, vol. 83, no. 1-2, pp. 419-432, 2016. 
[15] M. Xiao, W. X. Zheng, G. Jiang, and J. Cao, "Stability and bifurcation of delayed fractional-order dual congestion control algorithms," IEEE Transactions on Automatic Control, vol. 62, no. 9, pp. 4819-4826, 2017.

[16] M. Xiao, W. X. Zheng, G. Jiang, and J. Cao, "Undamped oscillations generated by Hopf bifurcations in fractional-order recurrent neural networks with caputo derivative," IEEE Transactions on Neural Networks and Learning Systems, vol. 26, no. 12, pp. 3201-3214, 2015.

[17] W. Ma, C. Li, Y. Wu, and Y. Wu, "Adaptive synchronization of fractional Neural Networks with unknown parameters and time delays," Entropy, vol. 16, no. 12, pp. 6286-6299, 2014.

[18] W. Y. Ma, C. P. Li, Y. J. Wu, and Y. Q. Wu, "Synchronization of fractional fuzzy cellular neural networks with interactions," Chaos, vol. 103, no. 10, Article ID 103106, 2017.

[19] G.-C. Wu, D. Baleanu, H.-P. Xie, and F.-L. Chen, "Chaos synchronization of fractional chaotic maps based on the stability condition," Physica A: Statistical Mechanics and Its Applications, vol. 460, pp. 374-383, 2016.

[20] J. Yu, C. Hu, and H. J. Jiang, " $\alpha$-stability and $\alpha$-synchronization for fractional-order neural networks," Neural Networks, vol. 35, pp. 82-87, 2012.

[21] X. J. Wu and H. T. Lu, "Outer synchronization between two different fractional-order general complex dynamical networks," Chinese Physics B, vol. 19, no. 7, Article ID 070511, 2010.

[22] L.-x. Yang and J. Jiang, "Adaptive synchronization of driveresponse fractional-order complex dynamical networks with uncertain parameters," Communications in Nonlinear Science and Numerical Simulation, vol. 19, no. 5, pp. 1496-1506, 2014.

[23] Y. Chai, L. Chen, R. Wu, and J. Sun, "Adaptive pinning synchronization in fractional-order complex dynamical networks," Physica A: Statistical Mechanics and Its Applications, vol. 391, no. 22, pp. 5746-5758, 2012.

[24] S. Dadras, S. Dadras, and H. R. Momeni, "Linear matrix inequality based fractional integral sliding-mode control of uncertain fractional-order nonlinear systems," Journal of Dynamic Systems, Measurement, and Control, vol. 139, no. 11, Article ID 111003, 2017.

[25] S. Shao, M. Chen, and X. Yan, "Adaptive sliding mode synchronization for a class of fractional-order chaotic systems with disturbance," Nonlinear Dynamics, vol. 83, no. 4, pp. 1855-1866, 2016.

[26] H. M. Liu, W. G. Sun, and G. Al-mahbashi, "Parameter identification based on lag synchronization via hybrid feedback control in uncertain drive-response dynamical networks," Advances in Difference Equations, vol. 2017, no. 1, p. 122, 2017.

[27] H. D. I. Abarbanel, N. F. Rulkov, and M. M. Sushchik, "Generalized synchronization of chaos: the auxiliary system approach," Physical Review E, vol. 53, no. 5, Article ID 4528, 1996.

[28] D. Ning, X. Q. Wu, J. A. Lu, and J. Lü, “Driving-based generalized synchronization in two-layer networks via pinning control," Chaos, vol. 25, no. 11, Article ID 113104, 2015.

[29] W. Sun, S. Wang, G. Wang, and Y. Wu, "Lag synchronization via pinning control between two coupled networks," Nonlinear Dynamics, vol. 79, no. 4, pp. 2659-2666, 2015.

[30] I. Podlubny, Fractional Differential Equations, Academic Press, San Diego, CA, USA, 1998.

[31] Q. Song and J. Cao, "On pinning synchronization of directed and undirected complex dynamical networks," IEEE Transactions on Circuits and Systems I: Regular Papers, vol. 57, no. 3, pp. 672-680, 2010.
[32] C. Li, W. Deng, and L. Zhao, "Well-posedness and numerical algorithm for the tempered fractional differential equations," Discrete \& Continuous Dynamical Systems - B, vol. 24, no. 4, pp. 1989-2015, 2019.

[33] Y. Li, Y. Chen, and I. Podlubny, "Mittag-Leffler stability of fractional order nonlinear dynamic systems," Automatica, vol. 45, no. 8, pp. 1965-1969, 2009.

[34] Y. Li, Y. Chen, and I. Podlubny, "Stability of fractional-order nonlinear dynamic systems: lyapunov direct method and generalized Mittag-Leffler stability," Computers \& Mathematics with Applications, vol. 59, no. 5, pp. 1810-1821, 2010.

[35] S. Zhang, Y. Yu, and H. Wang, "Mittag-Leffler stability of fractional-order Hopfield neural networks," Nonlinear Analysis: Hybrid Systems, vol. 16, pp. 104-121, 2015.

[36] S. Dadras, S. Dadras, H. Malek, and Y. Q. Chen, "A note on the lyapunov stability of fractional-order nonlinear systems," in Proceedings of the ASME 2017 International Design Engineering Technical Conferences and Computers and Information in Engineering Conference, American Society of Mechanical Engineers (ASME), Cleveland, OH, USA, 2017.

[37] W. Deng, "Numerical algorithm for the time fractional Fokker-Planck equation," Journal of Computational Physics, vol. 227, no. 2, pp. 1510-1522, 2007.

[38] J. Deng, L. Zhao, and Y. Wu, "Fast predictor-corrector approach for the tempered fractional differential equations," Numerical Algorithms, vol. 74, no. 3, pp. 717-754, 2017.

[39] H.-K. Chen and C.-I. Lee, "Anti-control of chaos in rigid body motion," Chaos, Solitons \& Fractals, vol. 21, no. 4, pp. 957-965, 2004. 


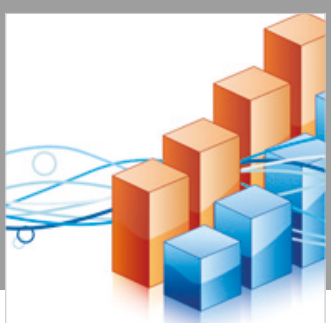

Advances in

Operations Research

\section{-n-m}
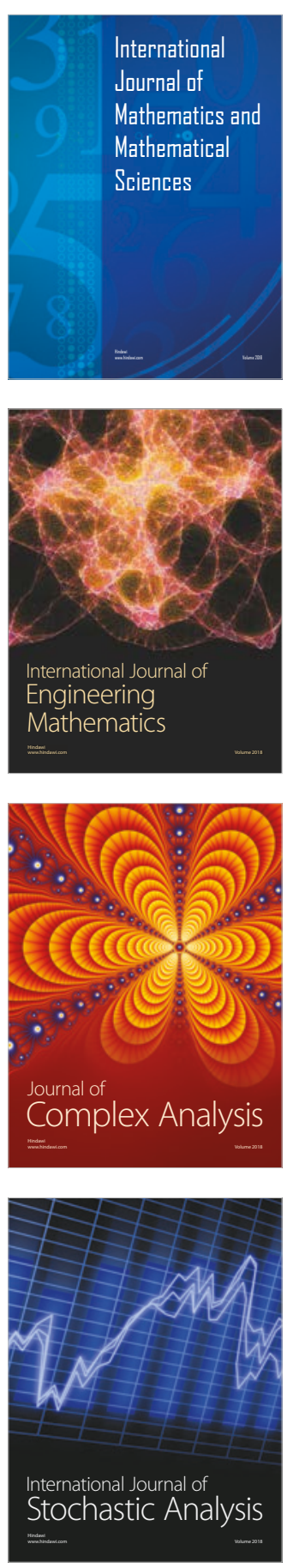
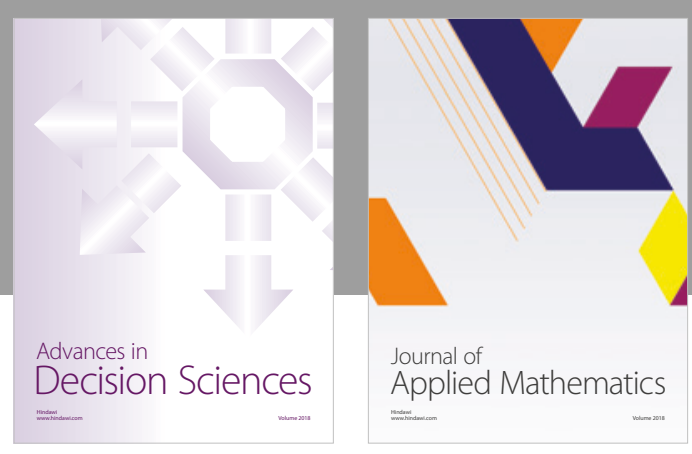

Journal of

Applied Mathematics
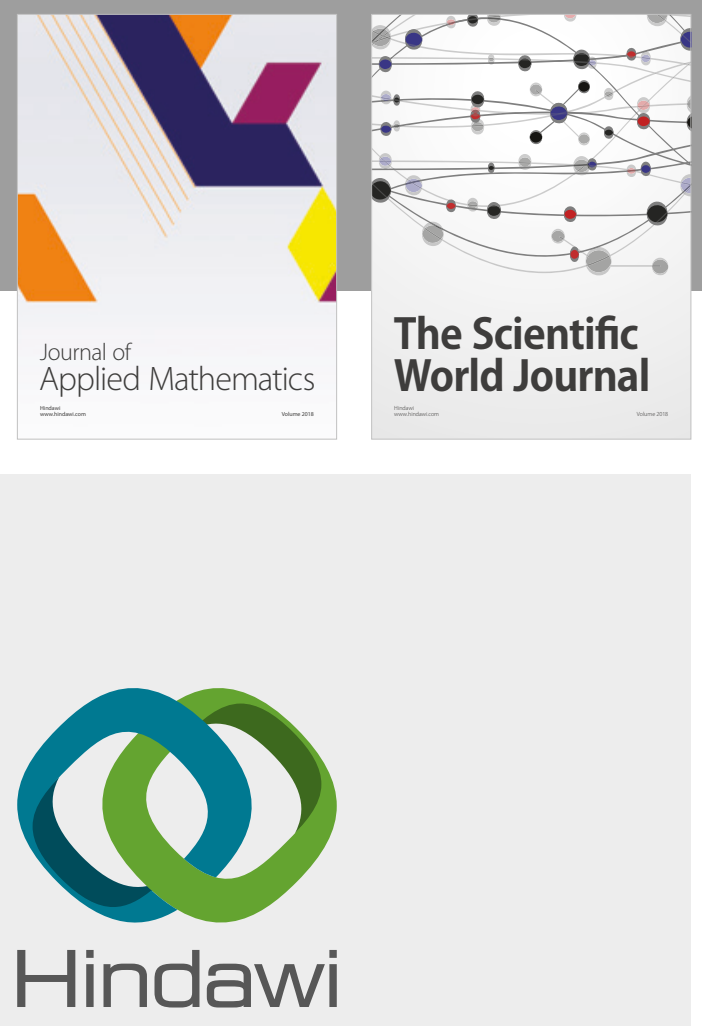

Submit your manuscripts at

www.hindawi.com

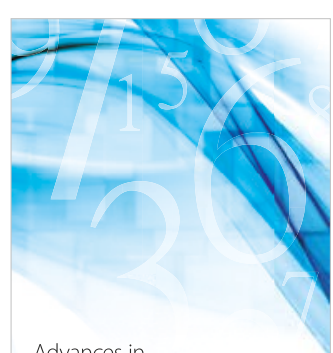

Advances in
Numerical Analysis
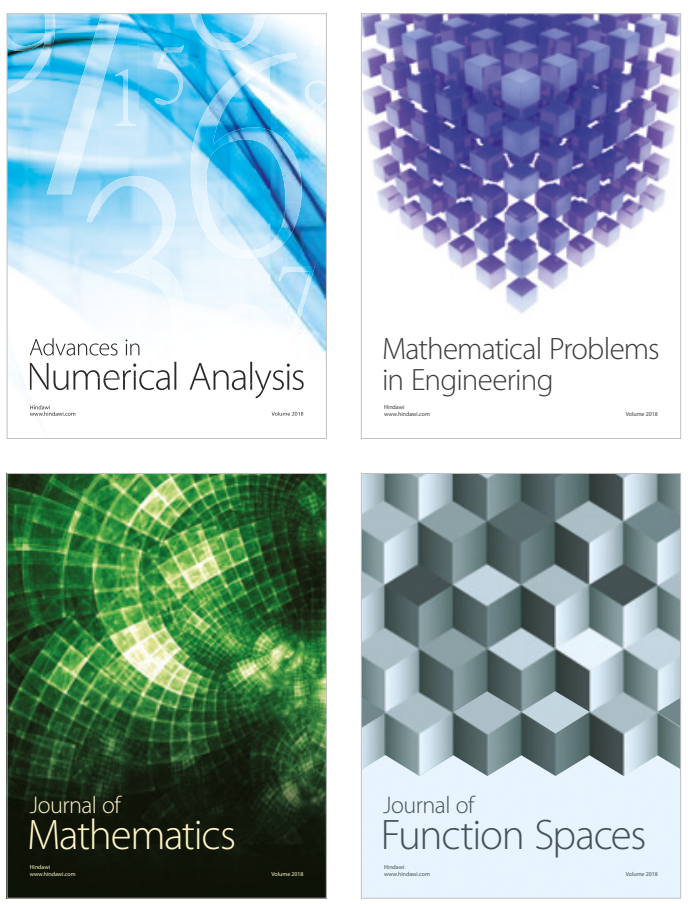

Mathematical Problems in Engineering

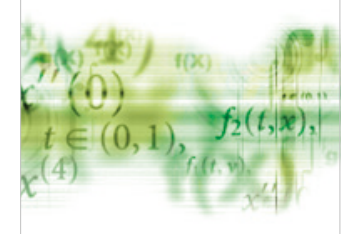

International Journal of

Differential Equations

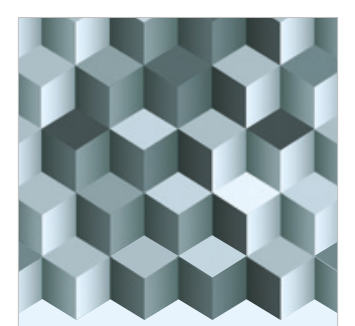

Journal of

Function Spaces
The Scientific

World Journal

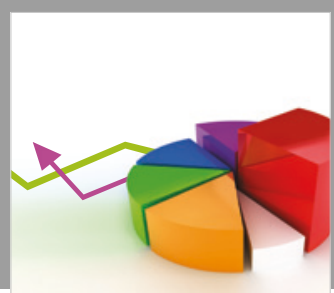

Journal of

Probability and Statistics
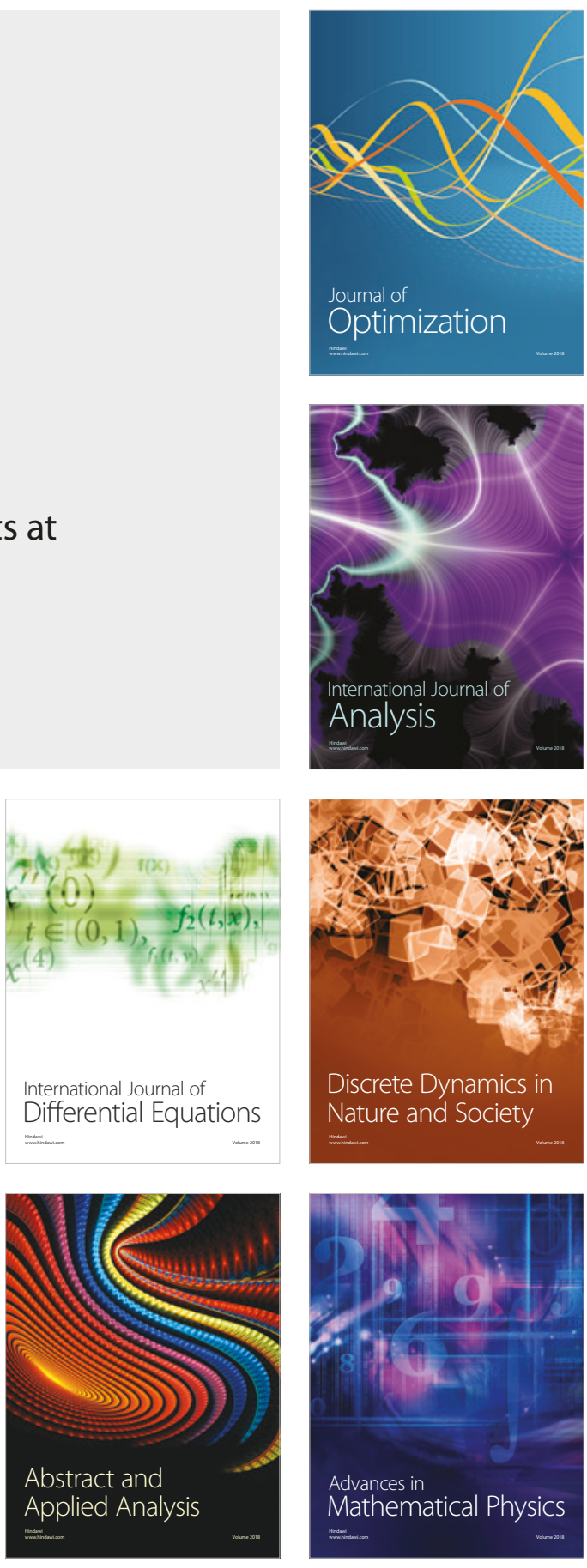